\title{
THE PROTECTIVE ROLE OF THE PERITROPHIC MEMBRANE IN THE TANNIN-TOLERANT LARVAE OF ORGYIA LEUCOSTIGMA (LEPIDOPTERA)
}

\author{
Raymond V. BarbehenN and Michael M. Martin* \\ Department of Biology, University of Michigan, Ann Arbor, MI 48109-1048, U.S.A.
}

(Received 3 April 1992; revised 15 June 1992)

\begin{abstract}
Tannic acid had no detrimental effect on the growth rates or digestion efficiencies of Orgyia leucostigma larvae. We examined three potential mechanisms which might allow these larvae to tolerate ingested tannic acid. (1) Little chemical modification of ingested tannic acid was found. Less than $10 \%$ of the tannic acid ingested by $O$. leucostigma was hydrolysed to gallic acid when measured with the rhodanine assay, and virtually all tannic acid was recovered in the frass when measured as total phenolics or with HPLC. (2) Peritrophic membranes were freely permeable to gallic acid but were impermeable to the higher molecular weight polyphenolic components of tannic acid. (3) Peritrophic membranes adsorbed less than $1 \%$ of the amount of tannic acid present in the guts of $O$. leucostigma larvae in vivo. Therefore, adsorption is not the means by which tannins are contained within the endoperitrophic space in $O$. leucostigma. We conclude that the peritrophic membrane acts as an ultrafilter. Further work is needed to determine whether ultrafiltration is dependent on tannin-binding substances in the gut fluid.
\end{abstract}

Key Word Index; Orgyia leucostigma; tannin; tannic acid; peritrophic membrane; herbivory

\section{INTRODUCTION}

Many species of phytophagous insects, including the subject of this study, Orgyia leucostigma (Lepidoptera: Lymantriidae), have been found to feed on tannin-containing diets without detrimental effects on digestion, growth and survival (Bernays, 1978; Berenbaum, 1983; Manuwoto and Scriber, 1986; Karowe, 1989). Several mechanisms have been proposed to account for tannin tolerance. In many insect species the potential adverse effects of tannins on digestion are avoided by gut conditions, especially alkalinity and detergency, that prevent the formation of complexes between tannins and digestive enzymes or food proteins (Berenbaum, 1980; Martin et al., 1987). It has also been proposed that hydrolysis in the gut is a process by which hydrolyzable tannins are detoxified in some species (Bernays, 1978) and that adsorption on the peritrophic membrane is a mechanism by which tannins are sequestered from the gut lumen (Bernays and Chamberlain, 1980). In this study we have examined three possible mechanisms of tannin-tolerance in late-instar $O$. leucostigma

*To whom all correspondence should be addressed. larvae: hydrolysis of tannic acid during digestion, ultrafiltration of tannic acid by the peritrophic membrane and adsorption of tannic acid on the peritrophic membrane.

\section{MATERLALS AND METHODS}

Effect of tannic acid on larval digestion and growth

$O$. leucostigma eggs were obtained from the Forest Pest Management Institute (Sault Ste. Marie, Ontario, Canada). Larvae were reared on Douglas fir tussock moth artificial diet (Bioserv) in an incubator with a $16 \mathrm{~h}$ light- $8 \mathrm{~h}$ dark photoperiod at $22^{\circ} \mathrm{C}$. Newly molted, final-instar male (fifth) and female (sixth) larvae were weighed to the nearest $0.1 \mathrm{mg}$ on a Sartorius electronic balance, placed in $30-\mathrm{ml}$ plastic cups, and assigned at random to control diet $(n=10)$, tannic acid diet $(n=10)$, or a group sacrificed to determine their percentage dry weight $(n=17)$. Mean fresh and dry weights of experimental larvae were $172.4 \pm 3.7$ (mean $\pm S E$ ) and $27.6 \pm 0.6$, respectively. Tannic acid diet was made by adding tannic acid to the rearing diet ( $5 \%$ dry weight, Sigma, lot No. 64F-0049) and the control diet contained an 
additional 5\% dry weight cellulose (Sigma). Both diets were prepared daily. Control and treatment larvae were supplied a weighed amount of artificial diet daily. Uneaten food, aliquots of artificial diet, sacrificed larvae and pupae were dried at $70^{\circ} \mathrm{C}$. Frass and artificial diet samples were freeze-dried daily and stored in a desiccator in the dark. Nutritional indices and relative growth and consumption rates were calculated on a dry weight basis (Waldbauer, 1968) using exponential mean weights of larvae (Montgomery, 1983).

\section{Chemical analyses}

Gallic acid. Gallic acid was measured using the rhodanine assay (Inoue and Hagerman, 1988). Samples $(30-60 \mathrm{mg}$ ) of ground lyophilized frass and artificial diet were extracted with $50 \%$ methanol $\left(2 \times 3 \mathrm{ml}, 50^{\circ} \mathrm{C}, 30 \mathrm{~min}\right)$ in a shaker. Combined extracts were centrifuged $(120 \mathrm{~g} ; 2 \mathrm{~min})$ and aliquots $(250 \mu \mathrm{l})$ of the supernatant solutions were mixed with $375 \mu \mathrm{l}$ of $0.667 \%$ methanolic rhodanine. After $5 \mathrm{~min}, 250 \mu 1$ of $0.5 \mathrm{M}$ potassium hydroxide was added, followed in $2.5 \mathrm{~min}$ by $5.375 \mathrm{ml}$ of distilled water. After 10-20 min the absorbance was measured at $520 \mathrm{~nm}$ with a Zeiss spectrophotometer. These values were corrected by subtracting absorbance due to rhodanine-positive substances in the control diet and in frass produced by larvae on the control diet. Net gallic acid production was calculated as (\% gallic acid in food $\times$ total amount consumed $)-(\%$ gallic acid in frass $\times$ total weight of frass produced). Recently purchased gallic acid (Sigma) was used as a standard. In order to express the amount of gallic acid produced by $O$. leucostigma as a percentage of the total gallic acid in ingested tannic acid (free and esterified), tannic acid $(1.0 \mathrm{mg} / \mathrm{ml})$ was completely hydrolyzed in $2.0 \mathrm{~N}$ sulfuric acid at $100^{\circ} \mathrm{C}$ for $26 \mathrm{~h}(n=3)$ and gallic acid in these hydrolysates was measured with the rhodanine assay. Hydrolyzed tannic acid was $77.0 \%$ gallic acid by weight.

Tannic acid. Tannic acid was measured using the Prussian blue assay for phenols (Price and Butler, 1977), substituting ferric ammonium sulfate for ferric chloride. Samples $(10-40 \mathrm{mg})$ of lyophilized artificial diets and frass were extracted and centrifuged as described for gallic acid analysis. Aliquots $(250 \mu 1)$ of supernatant solutions were mixed with $24 \mathrm{ml}$ of distilled water, after which $1.0 \mathrm{ml}$ of $0.1 \mathrm{M}$ ferric ammonium sulfate in $0.1 \mathrm{~N}$ hydrochloric acid and $1.0 \mathrm{ml}$ of $8 \mathrm{mM}$ potassium ferricyanide were added. After $10 \mathrm{~min}$ the absorbance of each solution was measured at $720 \mathrm{~nm}$. Tannic acid (Sigma; lot No. 64F-0049) was used as a standard. In separate experiments it was established that $93 \%$ of the tannic acid was recovered from freshly-prepared and freeze-dried diets using this method. Tannic acid budgets were calculated for each larva as $(\%$ tannic acid in frass $\times$ total dry weight of frass $) /(\%$ tannic acid in food $\times$ total dry weight ingested). Interfering (colorforming) substances in control diet and in frass from control larvae were quantified in order to correct tannic acid budgets. Tannic acid budgets were also corrected for gallic acid found in frass (measured with the rhodanine assay) by measuring the absorbance of gallic acid with the Prussian blue assay, calculating the tannic acid-equivalent of gallic acid produced by each larva, and subtracting these amounts from the levels of tannic acid in the frass of each larva.

HPLC analyses. HPLC analyses were conducted on extracts of lyophilized food and frass samples that had been stored in a desiccator in the dark at room temperature for 8 months. Samples $(3 \mathrm{mg})$ were extracted in $70 \%$ acetone $\left(2 \times 1.5 \mathrm{ml}, 50^{\circ} \mathrm{C}\right)$. Extracts were combined and evaporated to less than $1 \mathrm{ml}$ under a stream of nitrogen and lyophilized. Lyophilized extracts and tannic acid standards (Sigma) were redissolved in $1 \mathrm{ml}$ of mobile phase $[20 \%$ aqueous acetonitrile $(\mathrm{v} / \mathrm{v})$ with $1 \%(\mathrm{v} / \mathrm{v})$ acetic acid] and filtered ( 0.45 micron Gelman Acrodisc). Samples were injected with a Shimadzu autoinjector and run with a flow rate of $1 \mathrm{ml} / \mathrm{min}$ through a Waters Bondapak C-18 column $(10 \mu \mathrm{m} ; 4.6 \times$ $250 \mathrm{~mm}$ ) and guard column. Peaks were detected with a Shimadzu SPD-6AV u.v.-visible spectrophotometric detector at $280 \mathrm{~nm}(0.002 \mathrm{AU})$ and integrated with a Shimadzu C-R4A Chromatopac. The areas of peaks from interfering substances found in the control diet and frass of a larvae fed this diet were subtracted from the chromatograms of tannic acid diet and frass from larvae fed this diet. Tannic acid budgets were calculated for each larva using peaks of phenolics of higher molecular weight than gallic acid. The mean percentage recovery of tannic acid from artificial diets was $93.7 \%$ (SE 1.6), indicating that there was no effect of storage on tannic acid content.

Permeability of the peritrophic membrane to gallic acid and tannic acid

O. leucostigma were reared at 15 or $25^{\circ} \mathrm{C}$ on a $16 \mathrm{~h}$ light $-8 \mathrm{~h}$ dark photoperiod. Newly molted, final-instar larvae were placed on diets with $5 \%$ (dry weight) gallic acid $(n=8)$ or tannic acid $(n=8)$ and allowed to feed for 2 days. Control larvae $(n=3)$ were fed artificial diet without added gallic acid or tannic acid. Larvae were chilled at $-20^{\circ} \mathrm{C}$ for $5 \mathrm{~min}$ and further anesthetized with ethyl acetate before dissection. The foreguts and hindguts of dissected larvae were ligated 
with silk sutures (size 6-0). The entire gut was cut free from the body and rinsed in distilled water. Small apertures were then cut through the gut wall to expose the peritrophic membrane by lifting the gut tissue free from the peritrophic membrane with forceps and cutting through the tissue with iridectomy scissors. Since peritrophic membranes in guts containing artificial diet retained a rigid shape around the food and gut contents flowing from punctures made during dissection were clearly visible, no high molecular weight marker was added to artificial diets. Two to four holes were cut in each gut, exposing a mean total peritrophic membranc area of $3.7 \mathrm{~mm}^{2}$ in larvae fed gallic acid diet and $8.8 \mathrm{~mm}^{2}$ in larvae fed tannic acid diet. Ligated, fenestrated guts were rinsed in three successive beakers of fructose-saline solution $(300 \mathrm{mM}$ fructose, $227 \mathrm{mM}$ potassium chloride, $3 \mathrm{mM}$ sodium chloride) for $30-60 \mathrm{~s}$ and incubated in $2 \mathrm{ml}$ of fructose-saline solution for $2 \mathrm{~h}$ at $25^{\circ} \mathrm{C}$ in a shaker bath. This solution approximated the ionic composition of some larval lepidopteran midguts (Giordana and Sacchi, 1978) and was found to adequately balance the osmotic pressure in midguts containing fructose solutions in an experiment described below. A $1.5-\mathrm{ml}$ aliquot of each incubating solution was lyophilized and then redissolved in $750 \mu \mathrm{l}$ of $20 \%$ aqueous acetonitrile with $1 \%$ acetic acid for HPLC analysis. Lyophilization of tannic acid in fructose-saline solutions had no effect on tannic acid recovery [Fig. 2(b)]. The total amount of gallic acid or tannic acid present in the guts of experimental larvae ( $n=5$ fifth- and sixth-instar larvae on each diet) was estimated by sacrificing larvae fed $5 \%$ gallic acid and tannic acid diets and measuring the dry weights of their gut contents.

To control for the possibility that tannic acid incorporated in artificial diet was adsorbed on diet components, we allowed newly-molted larvae starved for $24-48 \mathrm{~h}$ to drink a solution containing purified tannic acid $(n=10)$. Tannic acid was purified on a Sephadex LH-20 column $(3 \times 40 \mathrm{~cm})$ according to Hagerman and Klucher (1986), with the exception that ascorbic acid was not added. Larvae were placed in Petri dishes with several 20- $\mu 1$ drops of a solution containing purified tannic acid $(1 \% \mathrm{w} / \mathrm{v})$, fructose $(0.83 \mathrm{M})$, fluorescein isothiocyanate-labelled dextran $\left(0.01 \%\right.$; Sigma; $\left.\mathrm{MW}=2 \times 10^{6}\right)$, and lysolecithin $(0.006 \%$; Sigma). Fructose was added as a feeding stimulant. FITC-labelled dextran was used as a high molecular weight marker to confirm the absence of damage to dissected midguts. Lysolecithin provided some degree of the detergency found in larval midguts. Control larvae $(n=2)$ were placed in dishes containing $20-\mu 1$ drops of a solution lacking tannic acid. Drinking times and approximate volumes imbibed were recorded for each larva. Thirty min after larvae drank $15-25 \mu$ l, they were dissected and the permeability of their peritrophic membranes was tested as described above. The fluorescence of the solutions in which gut preparations were incubated was measured with a Turner fluorometer.

\section{Adsorption of tannic acid by peritrophic membranes}

In vitro. Larvae were reared at $25^{\circ} \mathrm{C}$ on a $16 \mathrm{~h}$ light-8 $\mathrm{h}$ dark photoperiod. Fifth- and sixth-instar o. leucostigma larvae were dissceted after chilling at $-20^{\circ} \mathrm{C}$ for approx. $5 \mathrm{~min}$. Intact peritrophic membranes were removed, opened lengthwise under $0.1 \mathrm{M}$ buffer (pH 9.5 CHES (2-[N-cyclohexylamino]ethanesulfonic acid) or $\mathrm{pH} 6.5$ phosphate) and the contents rinsed out. Peritrophic membranes were rinsed in three successive beakers of buffer and stored in buffer until sufficient numbers were dissected. Peritrophic membranes were drained for $10 \mathrm{~s}$ on filter paper and weighed to the nearest $0.01 \mathrm{mg}$ on a Cahn 25 Electrobalance or a Sartorius electronic balance. Pairs of peritrophic membranes were matched by weight and randomly assigned to buffer containing tannic acid or to control buffer. The $\mathrm{pH}$ values of $5 \%(\mathrm{w} / \mathrm{v})$ tannic acid solutions were measured with a $\mathrm{pH}$ meter and adjusted to $\mathrm{pH} 6.5$ or 9.5 by addition of sodium hydroxide. Ascorbic acid $(60 \mathrm{mM})$ was added to $\mathrm{pH} 9.5$ buffers in two experiments to decrease the potential for tannic acid oxidation. Peritrophic membranes were incubated in buffer containing 5\% tannic acid for $30 \mathrm{~min}$ under nitrogen, following which they were returned to their original vials of control buffer and rinsed by occasional mixing for $15 \mathrm{~min}$. Alternatively, peritrophic membranes were rinsed for $30 \mathrm{~s}$. Control peritrophic membranes were incubated in $1.0 \mathrm{ml}$ of control buffer. Following incubation, peritrophic membranes were placed individually on preweighed pieces of aluminum foil and dried at $70^{\circ} \mathrm{C}$. Regressions of control peritrophic membrane dry weight on fresh weight were computed and used to calculate the initial dry weights of treated peritrophic membranes. The percentage of peritrophic membrane final weight contributed by adsorbed tannic acid was measured as (final peritrophic membrane dry weight - initial peritrophic membrane dry weight)/final peritrophic membrane dry weight. Changes in control and treated peritrophic membrane dry weights were compared by Mann-Whitney $U$-tests.

In vivo. Larvae were reared at $25^{\circ} \mathrm{C}$ on a $16 \mathrm{~h}$ light $-8 \mathrm{~h}$ dark photoperiod until the fifth instar and then fed $5 \%$ tannic acid (Sigma) diet at $20^{\circ} \mathrm{C}$ for 
Table 1. Consumption, digestion and growth in $O$. leucostigma larvae fed control diet or diet with 5\% tannic acid (mean \pm SE)

\begin{tabular}{lcc}
\hline & $\begin{array}{c}\text { Control } \\
\text { diet }\end{array}$ & $\begin{array}{c}\text { Tannic acid } \\
\text { diet }\end{array}$ \\
\hline RCR $^{\mathrm{s}}$ & $0.946 \pm 0.042$ & $0.892 \pm 0.035$ \\
$\mathrm{AD}^{*}$ & $44.3 \pm 0.8$ & $46.3 \pm 0.6$ \\
ECI & $19.7 \pm 0.7$ & $19.8 \pm 0.6$ \\
ECD & $44.4 \pm 1.3$ & $42.8 \pm 1.4$ \\
RGR & $0.185 \pm 0.007$ & $0.176 \pm 0.009$ \\
\hline
\end{tabular}

$N=10$ for control and tannic acid diets.

' RCR, relative consumption rate; RGR, relative growth rate; $A D$, approximate digestibility; ECI, efficiency of conversion of ingested food; ECD, efficiency of conversion of digested food.

$* P<0.05$ (Mann-Whitney U-test)

3 days. Intact peritrophic membranes containing the food in the midgut were dissected and placed on weighed pieces of aluminum foil $(n=15)$. Peritrophic membranes were separated from the midgut contents and rinsed for $10-15 \mathrm{~s}$ in distilled water. Midgut contents were dried at $60^{\circ} \mathrm{C}$. Fresh weights were measured as described above and each peritrophic membrane was extracted in $70 \%$ acetone $\left(2.5 \mathrm{ml}, 50^{\circ} \mathrm{C}, 30 \mathrm{~min}\right)$. Extracted peritrophic membranes were placed on weighed pieces of aluminum foil and dried at $6^{\circ} \mathrm{C}$. Acetone extracts were partially evaporated under a stream of nitrogen, lyophilized, and analyzed with HPLC as described above, with the exception that $50-\mu 1$ samples and standards were injected. Peaks corresponding to compounds previously found in artificial diet and insect tissues were subtracted from the total peak area. Adsorbed tannic acid (mg) was calculated from a tannic acid standard curve. The amount of tannic acid contained in each midgut was calculated as $5 \%$ of the dry weight of the midgut contents. Peritrophic membrane dry weights were found to be less than one-tenth of those in previous experiments, suggesting that $70 \%$ acetone extracted some peritrophic membrane component(s). Therefore, peritrophic membrane dry weights were calculated from their fresh weights using the percentage dry weight of peritrophic membranes found in an in vitro experiment (pH 6.5 control group; dry weight $=5.4 \% \times$ fresh weight).

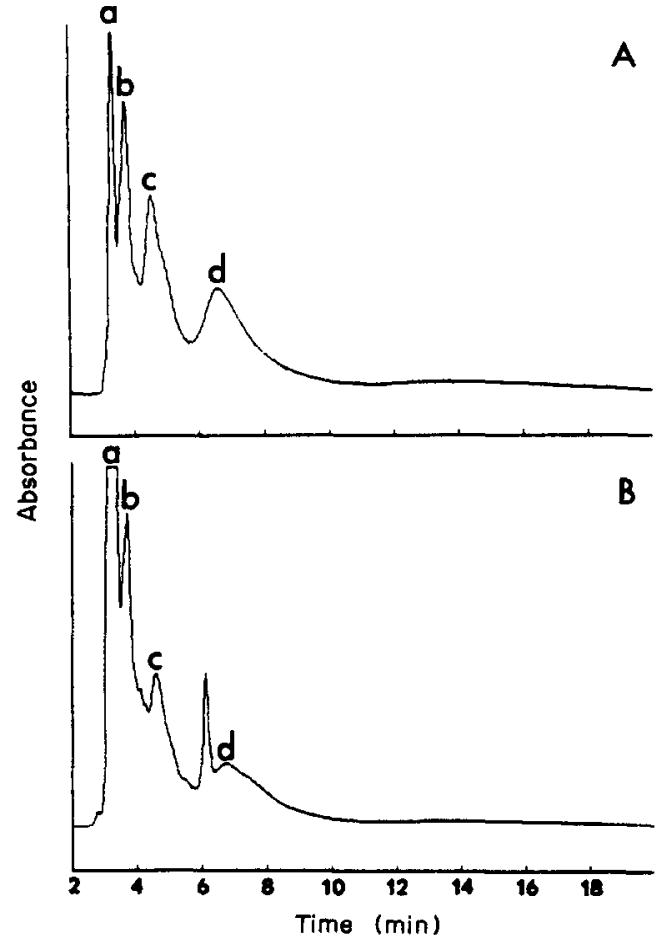

Fig. 1. (A) A chromatogram (HPLC) of tannic acid (Sigma). (B) A representative chromatogram of $70 \%$ acetone extract of frass from 0 . leucostigma fed $5 \%$ tannic acid diet. Peak $a$ is gallic acid (and other compounds in B). Peaks b-d are galloyl esters of glucose. The leading peak on peak $d$ in (B) was identified in frass from larvae fed the control diet also. No compounds eluted before $2 \mathrm{~min}$. Samples were chromatographed on a reverse-phase C-18 column with a $20 \%$ acetonitrile $/ 1 \%$ acetic acid aqueous mobile phase.

\section{RESULTS}

Relative consumption rate (RCR), relative growth rate (RGR), efficiency of conversion of ingested material (ECI), and efficiency of conversion of digested material (ECD) by $O$. leucostigma larvae were not significantly affected by the incorporation of tannic acid in their diet at $5 \%$ dry weight (Table 1). Approximate digestibilities (AD) of larvae fed diet containing tannic acid were significantly higher than those of larvae fed the control diet, albeit only by a small amount $(4.5 \%)$.

Using three different experimental approaches, we established that $90-100 \%$ of the tannic acid ingested by $O$. leucostigma larvae passes through the

Table 2. Amounts of tannic acid (TA) and gallic acid (GA) excreted by final-instar $O$. leucostigma larvae $(n=10)$

\begin{tabular}{|c|c|c|c|c|c|}
\hline \multirow[b]{2}{*}{ Method } & \multicolumn{2}{|c|}{$\begin{array}{l}\text { Ingested } \\
\text { (mg/larva) }\end{array}$} & \multicolumn{2}{|c|}{$\begin{array}{l}\text { Excreted } \\
\text { (mg/larva) }\end{array}$} & \multirow{2}{*}{$\begin{array}{c}\text { Ingested TA } \\
\text { aocounted for } \\
\text { in Frass } \\
(\% \pm \mathrm{SE})\end{array}$} \\
\hline & TA & GA & TA & GA & \\
\hline $\begin{array}{l}\text { HPLC } \\
\text { Prussian blue } \\
\text { Rhodanine }\end{array}$ & $\begin{array}{r}13.7 \\
9.3 \\
-\end{array}$ & $\overline{-}$ & $\begin{array}{r}14.1 \\
9.5 \\
-\end{array}$ & $\overline{7}$ & $\begin{array}{r}103.6 \pm 1.4 \\
101.3 \pm 1.3 \\
90.4 \pm 0.6\end{array}$ \\
\hline
\end{tabular}

*Assuming tannic acid not hydrolyzed to gallic acid remained as tannic acid. 


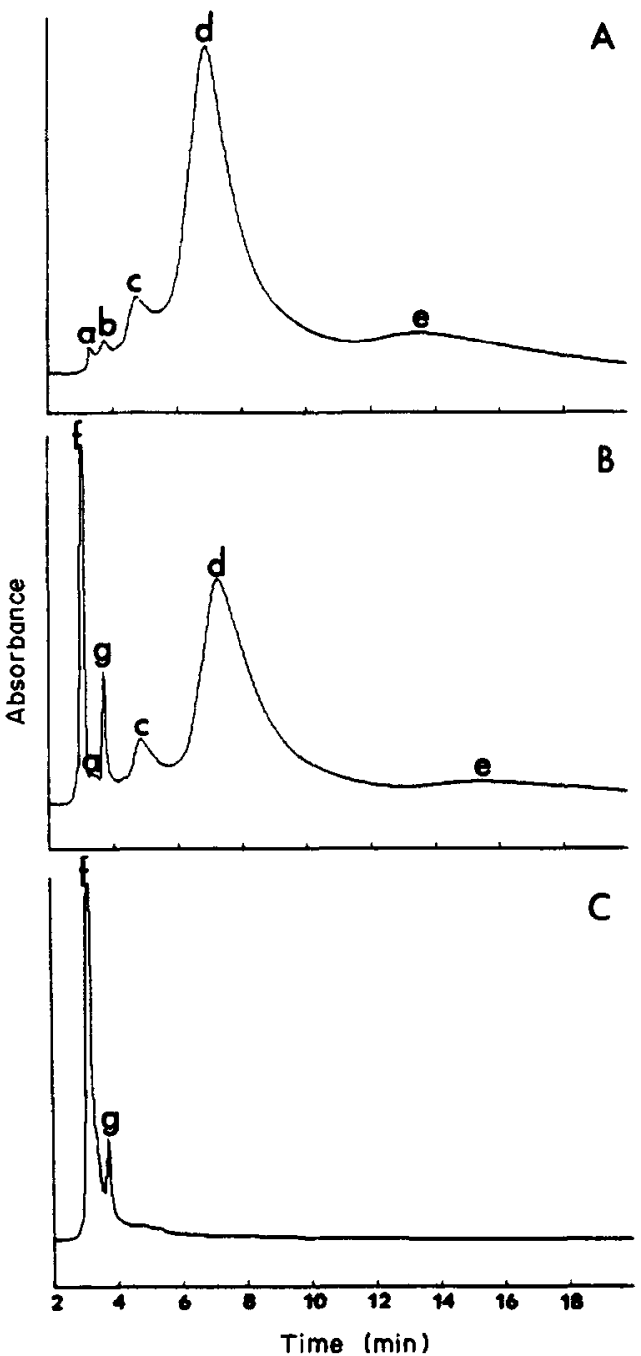

Fig. 2. (A) A chromatogram (HPLC) of purified tannic acid (Sigma). (B) A chromatogram of purified tannic acid in fructose-saline solution. (C) A representative chromatogram of fructose-saline solution in which an $O$. leucostigma gut with an exposed peritrophic membrane containing $1 \%$ purified tannic acid solution was incubated. No compounds eluted before 2 min. Peak a is gallic acid. Peaks b-e are galloyl esters of glucose. Peaks $f$ and $g$ are fructose-saline solution components.

gut unchanged (Table 2). All of the tannic acid ingested (assayed either directly using HPLC, or as phenol equivalents using the Prussian blue assay)

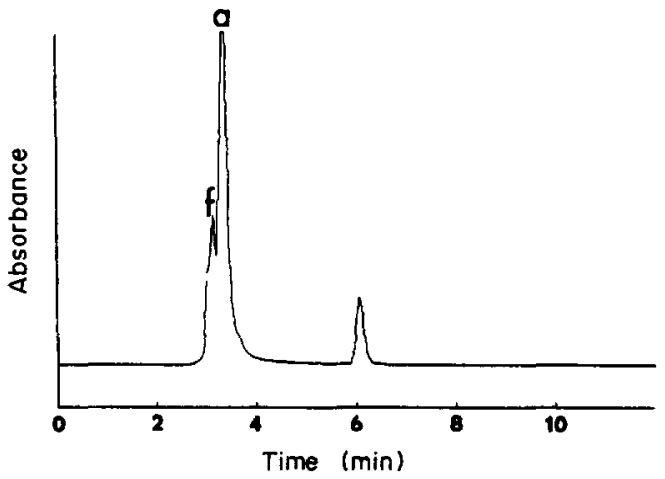

Fig. 3. A representative chromatogram (HPLC) of fructose-saline solution in which an $O$. leucostigma gut with an exposed peritrophic membrane containing $5 \%$ gallic acid diet was incubated. Peak a is gallic acid. $f$ is a fructose-saline solution component. The unidentified peak was also present in chromatograms from control larvae.

was recovered in the frass. By assaying the gallic acid content of food and frass (using the rhodanine assay), we established that more gallic acid was excreted than was ingested, suggesting limited hydrolysis of tannic acid in the gut. The amount of gallic acid detected in the frass can be fully accounted for by the hydrolysis of no more than $9.6 \%$ (SE 0.6 ) of the tannic acid ingested. Collectively, these three sets of experiments establish that chemical modification of ingested tannic acid in the gut cannot explain the tolerance of $O$. leucostigma larvae to dietary tannic acid.

Commercial tannic acid is primarily composed of galloyl esters of glucose along with some unesterified gallic acid (Hagerman et al., 1992). A chromatogram of the unpurified tannic acid used in this study is illustrated in Fig. 1(A). Peak a is gallic acid. Peaks b-e are galloyl glucose which differ in the number of galloyl groups attached to the glucose moiety. Comparison of the chromatograms of the tannic acid added to the diet [Fig. 1(A)] with a representative chromatogram of the $70 \%$ acetone extract of frass from 0 . leucostigma fed tannic acid [Fig. 1(B)] confirms the lack of chemical modification during passage through the digestive tract. Peaks which were not identified as tannic acid components (e.g. the

Table 3. Adsorption of tannic acid (TA) on $O$. leucostigma peritrophic membranes (PM) from a $5 \%$ tannic acid solution (mean $\pm \mathrm{SE}$ )

\begin{tabular}{lcccccc}
\hline & Ascorbic & \multicolumn{2}{c}{ Dry wt of PM (mg) } & & TA adsorbed & \\
\cline { 2 - 4 } pH & Acid & Initial & Final & & (mg/mg DW) $(n)$ & $P^{\text {a }}$ \\
\hline 6.5 & - & $0.33 \pm 0.03$ & $0.41 \pm 0.06$ & & $0.09 \pm 0.08(10)$ & 0.139 \\
9.5 & - & $0.54 \pm 0.02$ & $0.72 \pm 0.08$ & $0.15 \pm 0.06(8)$ & 0.034 \\
$9.5^{b}$ & + & $0.74 \pm 0.09$ & $1.03 \pm 0.05$ & $0.22 \pm 0.04(10)$ & 0.010 \\
\hline
\end{tabular}

"Probability of a significant difference between control and tannic acid-treated peritrophic membrane dry weight (Mann-Whitney U-test).

${ }^{b}$ PMs were rinsed for $30 \mathrm{~s}$ following incubation in $5 \%$ tannic acid. In the two other experiments peritrophic membranes were rinsed for $15 \mathrm{~min}$. 
Table 4. Adsorption of tannic acid (TA) on O. leucostigma peritrophic membranes (PM) in vivo when larvae were fed a $5 \% \mathrm{TA}$ diet (mean $\pm \mathrm{SE} ; n=15$ )

\begin{tabular}{lccc}
\hline $\begin{array}{l}\text { Dry wt of } \\
\text { PM }(\mathrm{mg})\end{array}$ & $\begin{array}{c}\text { TA in midgut } \\
(\mathrm{mg})\end{array}$ & $\begin{array}{c}\text { TA adsorbed on PM } \\
(\mathrm{mg} / \mathrm{mg} \mathrm{DW})\end{array}$ & $\begin{array}{c}\text { TA in midgut } \\
\text { adsorbed on PM } \\
(\%)\end{array}$ \\
\hline $0.48 \pm 0.07$ & $0.15 \pm 0.02$ & $0.002 \pm 0.0004$ & $0.56 \pm 0.10$ \\
\hline
\end{tabular}

leading peak on d) also appear in the extracts of frass and larvae fed a tannin-free diet.

In order to assess the capacity of the peritrophic membrane to act as a barrier to the diffusion of tannic acid from the endoperitrophic space, we tested for the presence of tannic acid components in the incubating solutions surrounding guts in which small holes had been cut to expose the peritrophic membrane. A chromatogram of purified tannic acid is illustrated in Fig. 2(A). Peaks $a$ and $b$ were not clearly separated in the presence of fructose-saline solution components [Fig. 2(B)], and may have been present in small amounts in incubating solutions, but none of the higher molecular weight components of tannic acid (peaks c-e) could be detected in these solutions after a $2 \mathrm{~h}$ incubation period [Fig. 2(C)]. The sensitivity of detection using HPLC was such that it would have been possible to measure the passage of as little as $0.75 \%$ of the total ingested tannic acid through the peritrophic membrane. Tannic acid in the gut was unable to diffuse through the peritrophic membrane whether it had been ingested in an agar-based artificial diet or in a simple aqueous solution containing tannic acid, fructose, and small amounts of lysolecithin and FITC-labelled dextran. Therefore, the lack of penetration of tannic acid through the peritrophic membrane was not due to adsorption of tannic acid on polymeric dietary components, e.g. protein or polysaccharides. Gallic acid (peak a) freely penetrated the peritrophic membrane (Fig. 3). We calculate that $23.2 \%$ (SE 3.1) of the gallic acid present in the gut penetrated the peritrophic membrane in $2 \mathrm{~h}$ through the small holes cut in the gut wall.

In order to assess the adsorptive capacity of the peritrophic membrane of $O$. leucostigma, we measured (1) the increase in weight of dissected peritrophic membranes following incubation in tannic acid solutions and (2) the amount of tannic acid adsorbed on peritrophic membranes in vivo. Incubation in $5 \%$ tannic acid solutions at $\mathrm{pH} 6.5$ or 9.5 resulted in increases in peritrophic membrane dry weight of $9-22 \%$, but in only two experiments were weight increases statistically significant (Table 3 ). The amount of tannic acid adsorbed in vivo was approx. $1 / 100$ of the amount adsorbed in vitro (Table 4). Thus, over $99 \%$ of ingested tannic acid appears to remain free in the lumen, and less than $1 \%$ is adsorbed on the peritrophic membrane in vivo. These experiments demonstrate that adsorption of tannic acid by the peritrophic membrane is not a mechanism that protects $O$. leucostigma larvae from the potential toxicity of this compound, and that adsorption cannot explain the retention of ingested tannic acid within the endoperitrophic space.

\section{DISCUSSION}

The finding that tannic acid has no detrimental effects on $O$. leucostigma larvae is in agreement with the earlier study of Karowe (1989). Our results suggest that protection from the ingested tannic acid in $O$. leucostigma is provided chiefly by a peritrophic membrane sheath that retains high molecular weight phenols in the endoperitrophic space. This mechanism for avoiding the potential toxicity of dietary tannins contrasts with the mechanisms found in some acridids, in which the major antitannin adaptation is bclieved to be hydrolysis of tannic acid to gallic acid. Over two-thirds of the tannic acid ingested by Schistocerca gregaria is hydrolyzed to gallic acid (Bernays, 1978). Our results show that less than $10 \%$ of ingested tannic acid is hydrolyzed to gallic acid by $O$. leucostigma larvae. Gallic acid, which may freely penetrate the peritrophic membrane of $O$. leucostigma, has been shown to have beneficial effects on the growth of other tree-feeding insects (Hamamura et al., 1966; Bernays, 1978; Bernays et al., 1983).

Previous studies of the effects of tannins on Lepidoptera and Orthoptera (Acrididae) have clearly implicated the importance of a peritrophic membrane that can serve as a barrier to tannic acid. Papilio polyxenes (Lepidoptera: Papilionidae) and graminivorous acridids with peritrophic membranes that do not retain tannins in the endoperitrophic space develop fatal lesions in their midgut epithelia (Bernays et al., 1980; Steinley and Berenbaum, 1985). By contrast, acridids with peritrophic membranes that do retain ingested tannins within the endoperitrophic space can tolerate high levels of tannins in their diets (Bernays et al., 1980).

It is possible to envision three distinct mechanisms by which the peritrophic membrane might act as a barrier to the passage of tannins: (1) by adsorption on 
the interior surface; (2) by ultrafiltration of unbound tannins and (3) by ultrafiltration of tannins that are bound to high molecular weight solute molecules, such as the proline-rich proteins produced by some vertebrates (Austin et al., 1989). Adsorption of tannins on the peritrophic membrane may contribute to tannin tolerance in acridids, but this mechanism appears to be insignificant in $O$. leucostigma. The peritrophic membranes of $S$. gregaria adsorb their own dry weight in tannic acid in vivo (Bernays and Chamberlain, 1980). Our results show that the peritrophic membranes of $O$. leucostigma adsorb less than $1 \%$ of their dry weight in vivo. We conclude, therefore, that in $O$. leucostigma the peritrophic membrane retains high molecular weight phenols within the endoperitrophic space by acting as an ultrafilter.

Neither peritrophic membrane thickness nor the presence of macromolecular food components in the gut appear to be important factors in affecting peritrophic membrane permeability in $O$. leucostigma. The peritrophic membrane in Lepidoptera is a sheath composed of multiple layers which are formed by delamination from the midgut epithelium as food passes through the gut. Therefore, impermeability to tannic acid in starved newly-molted larvae suggests that peritrophic membrane pore size is sufficiently small to obstruct passage by tannic acid even when the peritrophic membrane is at its thinnest. Peritrophic membranes of larvae with empty guts were as impermeable to tannic acid as peritrophic membranes of larvae with guts filled with artificial diet.

In studies of several species of Diptera and Lepidoptera, it has been demonstrated that peritrophic membranes are freely permeable to low molecular weight molecules, proteins and dextrans, and small particulates (Zhuzhikov, 1970; Adang and Spence, 1983; Terra and Ferreira, 1983; Espinoza-Fuentes et al., 1984; Peters and Wiese, 1986; Santos and Terra, 1986; Wolfersberger et al., 1986; Zimmerman and Peters, 1987). Pore diameters in larval Diptera have been estimated to range from 1 to $9 \mathrm{~nm}$, depending on the method used (Zhuzhikov, 1970; Terra and Ferreira, 1983; Peters and Wiese, 1986). In larval Lepidoptera pore diameters have generally been estimated to range between $7-8 \mathrm{~nm}$ (Santos and Terra, 1986; Wolfersberger et al., 1986), with the exception of $O$. pseudotsugata, in which Adang and Spence (1983) report that pore sizes range from 300 to $800 \mathrm{~nm}$. Using a relationship between molecular weight and molecular dimensions derived from several non-proteinacous compounds (Lehninger, 1975; Freifelder, 1987), we calculate that the major high-molecular weight components of tannic acid [789-1027 kDa; (Hagerman et al., 1992)], range from approx. $3.5-4.7 \mathrm{~nm}$ in diameter. Unless the pores of the peritrophic membranes of tanninadapted Lepidoptera are significantly smaller than the pores of the species studied to date, it seems unlikely that the peritrophic membrane could serve as an effective ultrafilter for unbound tannins. These considerations leave ultrafiltration of bound tannins as the most likely explanation for the capacity of the peritrophic membrane to contain ingested tannins within the endoperitrophic space.

We hasten to add, however, that this study provides no direct evidence for the presence in the gut fluid of $O$. leucostigma larvae of insect-derived tannin-binding macromolecules. To the best of our knowledge, the production of tannin-binding proteins by herbivorous insects has not been examined. Comparative studies of the production of tanninbinding substances, pore sizes and ultrafiltration properties of peritrophic membranes are likely to generate important insights into the anti-tannin adaptations of herbivorous insects.

Acknowledgements - This study was supported by NSF Grant BSR-8904043 to MMM. We thank Ann E. Hagerman for her comments and help with methods for measuring tannic acid components.

\section{REFERENCES}

Adang M. J. and Spence K. D. (1983) Permeability of the peritrophic membrane of the douglas fir tussock moth (Orgyia pseudotsugata). Comp. Biochem. Physiol. 75A, 233-238.

Austin P. J., Suchar L. A., Robbins C. T. and Hagerman A. E. (1989) Tannin binding proteins in the saliva of deer and their absence in the saliva of sheep and cattle. J. chem. Ecol. 15, 1335-1347.

Berenbaum M. (1980) Adaptive significance of midgut $\mathrm{pH}$ in larval Lepidoptera. Am. Nat. 115, 138-146.

Berenbaum M. (1983) Effect of tannins on growth and digestion in two species of papilionids. Entomologia exp. appl. 34, 245-250.

Bernays E. A. (1978) Tannins: an alternative viewpoint. Entomologia exp. appl. 24, 244-253.

Bernays E. A. and Chamberlain D. J. (1980) A study of tolerance of ingested tannin in Schistocerca gregaria. J. Insect Physiol. 26, 415 420.

Bernays E. A., Chamberlain D. and McCarthy P. (1980) The differential effects of ingested tannic acid on different species of acridoidea. Entomologia exp. appl. 28, 158-166.

Bernays E. A., Chamberlain D. J. and Woodhead S. (1983) Phenols as nutrients for a phytophagous insect Anacridium melanorhodon. J. Insect Physiol. 29, 535-539.

Espinoza-Fuentes F. P., Ferreira C. and Terra W. R. (1984) Spatial organization of digestion in the larval and imaginal stages of the sciarid fly Trichosia pubescens. Insect Biochem. 14, 631-638.

Freifelder D. (1987) Molecular Biology. Jones and Bartlett Publishers, Boston, MA.

Giordana B. and Sacchi F. (1978) Cellular ionic concentrations in the midgut of two larvae of Lepidoptera in vivo and in vitro. Comp. Biochem. Physiol. 59A, 17-20. 
Hagerman A. E., Robbins C. T., Weerasuriya Y., Wilson T. C. and McArthur C. (1992) Tannin chemistry in relation to digestion. J. Range Mangmt 45, 57-62.

Hamamura Y., Kuwata K. and Masuda H. (1966) Effect of gallic acid on the growth of the silkworm larvae Bombyx mori L. Nature 212, 1386-1387.

Inoue K. H. and Hagerman A. E. (1988) Determination of gallotannin with rhodanine. Analyt. Biochem. 169, 363-369.

Karowe D. (1989) Differential effect of tannic acid on two tree-feeding Lepidoptera: implications for theories of plant-herbivore chemistry. Oecologia 80, 507-512.

Lehninger A. L. (1975) Biochemistry. Worth, New York.

Manuwoto S. and Scriber J. M. (1986) Effects of hydrolyzable and condensed tannin on growth and development of two species of polyphagous Lepidoptera: Spodoptera eridania and Callosamia promethea. Oecologia 69. 225-230.

Martin J. S., Martin M. M., Bernays E. A. (1987) Failure of tannic acid to inhibit digestion or reduce digestibility of a plant protein in the gut fluids of insect herbivores: implications for theories of plant defense. J. chem. Ecol. 13, 605-621.

Montgomery M. E. (1983) Biomass and nitrogen budgets during larval development of Lymantria dispar and Choristoneura fumiferana: allometric relationships. In Proc. Forest Defoliator-Host Interactions (Eds Talerico R. and Montgomery M.), pp. 133-140. USDA Forest Service NE Station General Technical Report NE-25.

Peters W. and Wiese B. (1986) Permeability of the peritrophic membranes of some Diptera to labeled dextrans. J. Insect Physiol. 32, 43-50.
Price M. L. and Butler L. G. (1977) Rapid visual estimation and spectrophotometric determination of tannin content of sorghum grain. J. Agric. Food Chem. 25, $1268-1273$.

Santos C. D. and Terra W. R. (1986) Distribution and characterization of oligomeric digestive enzymes from Erinnyis ello larvae and inferences concerning secretory mechanisms and the permeability of the peritrophic membrane. Insect Biochem. 16, 691-700.

Steinly B. A. and Berenbaum M. (1985) Histopathological effects of tannins on the midgut epithelium of Papilio polyxenes and Papilio glaucus. Entomologia exp. appl. $39,3-9$.

Terra W. R. and Ferreira C. (1983) Further evidence that enzymes involved in the final stages of digestion by Rhynchosciara do not enter the endoperitrophic space. Insect Biochem. 13, 143-150.

Waldbauer G. P. (1968) The consumption and utilization of food by insects. Adv. Insect Physiol. 5, 229-289.

Wolfersberger M. G., Spaeth D. D. and Dow J. A. T. (1986) Permeability of the peritrophic membrane of tobacco hornworm larval midgut. Am. Zool. 26, $74 \mathrm{~A}$.

Zhuzhikov D. P. (1970) Permeability of the peritrophic membrane in larvae of Aedes aegypti. J. Insect Physiol. 16, 1193-1202.

Zimmerman D. and Peters W. (1987) Fine structure and permeability of peritrophic membranes of Calliphora erythrocephala (Meigan) (Insecta: Diptera) after inhibition of chitin and protein synthesis. Comp. Biochem. Physiol. 86B, 353-360. 